TRANSACTIONS OF THE

AMERICAN MATHEMATICAL SOCIETY

Volume 355, Number 7 , Pages 2715-2732

S 0002-9947(03)03200-8

Article electronically published on March 19, 2003

\title{
ON THE CANONICAL RINGS OF COVERS OF SURFACES OF MINIMAL DEGREE
}

\author{
FRANCISCO JAVIER GALLEGO AND BANGERE P. PURNAPRAJNA
}

\begin{abstract}
In one of the main results of this paper, we find the degrees of the generators of the canonical ring of a regular algebraic surface $X$ of general type defined over a field of characteristic 0 , under the hypothesis that the canonical divisor of $X$ determines a morphism $\varphi$ from $X$ to a surface of minimal degree $Y$. As a corollary of our results and results of Ciliberto and Green, we obtain a necessary and sufficient condition for the canonical ring of $X$ to be generated in degree less than or equal to 2 . We construct new examples of surfaces satisfying the hypothesis of our theorem and prove results which show that many a priori plausible examples cannot exist. Our methods are to exploit the $\mathcal{O}_{Y}$-algebra structure on $\varphi_{*} \mathcal{O}_{X}$. These methods have other applications, including those on Calabi-Yau threefolds. We prove new results on homogeneous rings associated to a polarized Calabi-Yau threefold and also prove some existence theorems for Calabi-Yau covers of threefolds of minimal degree. These have consequences towards constructing new examples of Calabi-Yau threefolds.
\end{abstract}

\section{INTRODUCTION}

The canonical models of surfaces of general type have attracted the attention of many geometers. The questions on projective normality and ring generators of the canonical ring are of particular interest. Kodaira $\mathrm{Kod}$ first proved that $\left|K_{X}^{\otimes m}\right|$ embeds a minimal surface of general type $X$ as a projectively normal variety for all $m \geq 8$. This was later improved by Bombieri [Bo, who proved the same result if $m \geq 6$, and by Ciliberto $\mathrm{Ci}$, who lowered the bound to $m \geq 5$. We proved in GP1 more general results on projective normality and higher syzygies for adjunction bundles for an algebraic surface. As a corollary of these results we recovered and improved the results of Bombieri and Ciliberto on projective normality, and extended them to higher syzygies. We also recovered and extended the results of Reid $[\underline{R}$ on the ring generators of the canonical ring of a surface of general type.

An important class of minimal surfaces of general type comprises those whose canonical divisor is base-point-free. Surfaces with base-point-free canonical divisor fall naturally into two categories corresponding to the division of curves of genus greater than one into hyperelliptic and non-hyperelliptic: those whose canonical

Received by the editors July 5, 2002.

2000 Mathematics Subject Classification. Primary 14J29.

The first author was partially supported by MCT project number BFM2000-0621 and by UCM project number PR52/00-8862. The second author was partially supported by the General Research Fund of the University of Kansas at Lawrence. The first author is grateful for the hospitality of the Department of Mathematics of the University of Kansas at Lawrence. 
morphism maps onto a surface of minimal degree and those whose canonical morphism does not map to such a surface. By a surface of minimal degree we mean a nondegenerate surface in projective space whose degree is equal to its codimension plus 1 . The surfaces of minimal degree are classically known: they are (linear) $\mathbf{P}^{2}$, the Veronese surface in $\mathbf{P}^{5}$, and smooth rational scrolls or cones over a rational normal curve (see $[\mathrm{EH}]$ ). Note that, even though we are drawing an analogy between hyperelliptic curves and surfaces of general type whose canonical morphism maps onto a surface of minimal degree, the theory is much harder for surfaces, because higher degree covers are involved. Examples of such covers are shown in Section 3.

In this paper we deal with surfaces of general type whose canonical morphism maps onto a surface of minimal degree. The surfaces whose canonical morphism does not map onto a surface of minimal degree have been studied by Ciliberto and Green (see [Ci] and G]). Green and Ciliberto proved the following beautiful result regarding the generators of the canonical ring:

Let $X$ be a regular surface of general type with base-point-free canonical divisor. Assume that the canonical morphism $\varphi$ satisfies the following conditions:

(1) $\varphi$ does not map $X$ generically 2: 1 onto the projective plane;

(2) $\varphi(X)$ is not a surface of minimal degree (other than linear $\mathbf{P}^{2}$ ).

Then the canonical ring of $X$ is generated in degree less than or equal to 2.

The surfaces of general type $X$ whose canonical morphism $\varphi$ maps $X$ onto a surface of minimal degree $Y$ have been studied by Horikawa (see [H1, H2], H3] and [H4]), Catanese [Ca], Konno [Kon, Mendes Lopes and Pardini [MP], among others, where they play a central role in the classification of surfaces of general type with small $c_{1}^{2}$ and in questions about degenerations and the moduli of surfaces of general type. The study of these surfaces has a direct bearing on the study of linear series on threefolds such as Calabi-Yau threefolds, as the results in [OP], and the authors' results in GP2 and Section 4 of this article show.

The study of the canonical rings of these surfaces is carried out in Section 2. We determine the precise degrees of the generators of its canonical ring (see Theorem 2.1). The answer depends on the degree of $\varphi$ and the degree of $Y$. As a corollary of our result and the result of Ciliberto and Green (see Corollary 2.8), we find that conditions (1) and (2) above characterize the regular surfaces of general type with base-point-free canonical bundle whose canonical ring is generated in degree less than or equal to 2 . This result is surprising, because it contrasts with the situation for higher-dimensional varieties, which, as we show in [GP3], differs from the situation for surfaces. Indeed, we show in [GP3] that there is no higher-dimensional analogue of Corollary 2.8, and therefore there is no converse of Green's result (cf. G], Theorem 3.9.3) for higher dimensional varieties of general type.

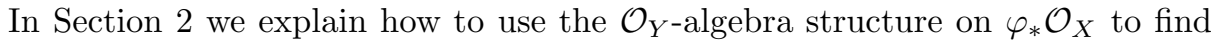
the multiplicative structure of the canonical ring of $X$. Even though we reduce the problem from a complicated variety to a simpler variety, as a surface $Y$ of minimal degree is, there are certain difficulties that arise in the process. The proof of Theorem 2.1 involves the study of multiplication of global sections of line bundles on a surface $X$ of general type. To do so we reduce the problem to $Y$, and this amounts to studying maps of multiplication of global sections of vector bundles instead of line bundles. This is the first difficulty. Moreover, the relation between the multiplication maps of global sections of line bundles on $X$ and the multiplication maps of global sections of vector bundles on $Y$ is governed by the 
$\mathcal{O}_{Y}$-algebra structure on $\varphi_{*} \mathcal{O}_{X}$. However, we prove our results for a large class of surfaces $X$. So their canonical morphism $\varphi$ might, and actually does, correspond to many, quite diverse $\mathcal{O}_{Y}$-algebra structures. This is in sharp contrast to hyperelliptic curves, where canonical morphisms correspond to degree 2 algebra structures, which are all quite similar and very easy to describe. The algebra structures arising from canonical morphisms of surfaces are much more complicated and harder to determine. This is the second difficulty one encounters, a difficulty that we are able to overcome in the context of this paper.

In Section 3 we construct new examples of surfaces of general type mapping to a surface of minimal degree and also recall some known ones. It is interesting to know in general what positive integers occur as degrees of the canonical morphism if the image is a surface of minimal degree. An answer to this question is helpful in finding new examples. Having this philosophy in mind, we prove results which show that some natural ways to construct examples do not work. For instance, it follows from the results in Section 3 that odd degree covers of smooth scrolls or cyclic covers of degree bigger than 3 of surfaces of minimal degree, induced by the canonical morphism, do not exist.

The results on surfaces of general type mentioned above have ramifications for Calabi-Yau threefolds. In Section 4 of this article we apply these results to obtain new results for a polarized Calabi-Yau threefold $(X, B)$ with $B$ a base-point-free and ample divisor. Among other things, we find out the degrees of the generators of the homogeneous ring associated to $B$, and we give a characterization of polarized Calabi-Yau threefolds $(X, B)$ whose associated homogeneous ring is generated in degree less than or equal to 2. The construction of examples of Calabi-Yau threefolds has evoked interest in recent years. One of the important sources of these examples is to take covers of threefolds of minimal degree. In Section 4, we prove some existence theorems for Calabi-Yau covers of threefolds of minimal degree. For instance, from these results, which are more general, it follows that a Calabi-Yau cover of prime degree greater than 3 induced by a complete linear series cannot come from a group action.

We will expand on these ideas in two forthcoming articles, GP3] and [GP5]. In the first we study the canonical ring of higher-dimensional varieties of general type whose canonical morphism maps onto a variety of minimal degree. One of the results in GP3] shows that the converse of the theorem of Ciliberto and Green for surfaces proved in this article is false for higher-dimensional varieties of general type. In the second we carry out a detailed study of homogeneous rings associated to line bundles on trigonal curves.

\section{Preliminaries}

Convention. Throughout this article we will work over an algebraically closed field of characteristic 0 .

In this section we will recall some known facts about the push-forward of the structure sheaf of a variety by a flat, finite morphism. We summarize these facts below, and refer for the proof to [HM], Section 2 .

Let $X$ and $Y$ be algebraic varieties. Let $\pi: X \longrightarrow Y$ be a finite, flat morphism of degree $n$. We have the following facts:

1.1. The sheaf $\pi_{*} \mathcal{O}_{X}$ is a rank $n$, locally free sheaf on $Y$ of algebras over $\mathcal{O}_{Y}$. 
1.2. There exists a map

$$
\frac{1}{n} \operatorname{tr}: \pi_{*} \mathcal{O}_{X} \longrightarrow \mathcal{O}_{Y}
$$

of sheaves of $\mathcal{O}_{Y}$-modules defined locally as follows: Given $\alpha \in \pi_{*} \mathcal{O}_{X}$, we consider the homomorphism of $\mathcal{O}_{Y}$-modules

$$
\pi_{*} \mathcal{O}_{X} \stackrel{\alpha}{\rightarrow} \pi_{*} \mathcal{O}_{X}
$$

induced by multiplication by $\alpha$. Then we define $\frac{1}{n} \operatorname{tr}(\alpha)$ as the trace of such a homomorphism divided by $n$.

1.3. $\frac{1}{n} \operatorname{tr}$ is surjective; in fact, the map $\mathcal{O}_{Y} \hookrightarrow \pi_{*} \mathcal{O}_{X}$ induced by $\pi$ is a section of $\frac{1}{n} \operatorname{tr}$. Therefore the sequence

$$
0 \longrightarrow E \longrightarrow \pi_{*} \mathcal{O}_{X} \stackrel{\frac{1}{n} \operatorname{tr}}{\longrightarrow} \mathcal{O}_{Y} \longrightarrow 0
$$

splits. $E$ is the kernel of $\frac{1}{n} \operatorname{tr}$ and locally consists of the trace 0 elements of $\pi_{*} \mathcal{O}_{X}$. We will call $E$ the trace-zero module of $\pi$.

1.4. $\pi_{*}\left(\mathcal{O}_{X}\right)$ is a sheaf of $\mathcal{O}_{Y}$-algebras; therefore, it has a multiplicative structure. Its multiplication map is an $\mathcal{O}_{Y}$-linear map

$$
\left[\mathcal{O}_{Y} \oplus E\right] \otimes\left[\mathcal{O}_{Y} \oplus E\right] \longrightarrow \mathcal{O}_{Y} \oplus E
$$

made of four components. The first component

$$
\mathcal{O}_{Y} \otimes \mathcal{O}_{Y} \longrightarrow \mathcal{O}_{Y} \oplus E
$$

is given by the multiplication in $\mathcal{O}_{Y}$, and therefore goes to $\mathcal{O}_{Y}$. The components

$$
\begin{gathered}
\mathcal{O}_{Y} \otimes E \longrightarrow \mathcal{O}_{Y} \oplus E, \\
E \otimes \mathcal{O}_{Y} \longrightarrow \mathcal{O}_{Y} \oplus E
\end{gathered}
$$

are given by the left and right module structure of $E$ over $\mathcal{O}_{Y}$, and therefore go to $E$. Finally, there is a fourth component

$$
E \otimes E \longrightarrow \mathcal{O}_{Y} \oplus E
$$

which factors through

$$
S^{2} E \longrightarrow \mathcal{O}_{Y} \oplus E,
$$

for multiplication in $\pi_{*} \mathcal{O}_{Y}$ is commutative.

\section{Covers of Surfaces of Minimal DEgREE}

Our purpose in this section is to study the generators of the canonical ring of certain surfaces of general type. Specifically, we are interested in studying those regular surfaces of general type whose canonical divisor is base-point-free and such that the image of the canonical morphism is a variety of minimal degree. We obtain the following result.

Theorem 2.1. Let $S$ be a regular surface of general type with at worst canonical singularities and such that its canonical bundle $K_{S}$ is base-point-free. Let $\varphi$ be the canonical morphism of $S$. Let $n$ be the degree of $\varphi$ and assume that the image of $\varphi$ is a surface of minimal degree $r$. Then:

1) if $n=2$ and $r=1$ (i.e., if $\varphi$ is generically $2: 1$ onto $\mathbf{P}^{2}$ ), then the canonical ring of $S$ is generated by its part of degree 1 and one generator in degree 4;

2) if $n \neq 2$ or $r \neq 1$, then the canonical ring of $S$ is generated by its part of degree $1, r(n-2)$ generators in degree 2 and $r-1$ generators in degree 3 . 
The knowledge of how many linearly independent generators are needed in each degree is obtained from the knowledge of the image of the multiplication maps of global sections of powers of the canonical bundle. We study those multiplication maps by studying similar maps of a curve $C$ in $\left|K_{S}\right|$. Thus we will first prove the following proposition.

Proposition 2.2. Let $C$ be a smooth, irreducible curve. Let $\theta$ be a base-point-free line bundle on $C$ such that $\theta^{\otimes 2}=K_{C}$. Let $\pi$ be the morphism induced by $|\theta|$, let $n$ be the degree of $\pi$, and assume that $\pi(C)$ is a rational normal curve of degree $r$. Let $\beta(s, t)$ be the multiplication map

$$
H^{0}\left(\theta^{\otimes s}\right) \otimes H^{0}\left(\theta^{\otimes t}\right) \longrightarrow H^{0}\left(\theta^{\otimes s+t}\right), \text { for all } s, t>0 .
$$

The codimension of the image of $\beta(s, t)$ in $H^{0}\left(\theta^{\otimes s+t}\right)$ is as follows:

a) If $r=1$, the codimension is:

a.1) $n-2$, for $s=t=1$,

a.2) 0 , for $s=2, t=1$, i.e., $\beta(2,1)$ surjects,

a.3) 1 , for $s=3, t=1$,

a.4) 1 , for $s=t=2, n=2$ and 0 if $n>2$,

a.5) 0 , for $s \geq 4, t=1$, i.e., $\beta(s, 1)$ surjects for all $s \geq 4$.

b) If $r>1$, the codimension is:

b.1) $r(n-2)$, for $s=t=1$,

b.2) $r-1$, for $s=2, t=1$,

b.3) 0 , for $s \geq 3, t=1$, i.e., $\beta(s, 1)$ surjects for all $s \geq 3$.

Moreover, if $r=1$ and $n=2$, then the image of $\beta(2,2)$ and the image of $\beta(3,1)$ are equal.

In order to prove Proposition 2.2, we will use the following.

Lemma 2.3. Let $C, \theta$ and $\pi$ be as in the statement of Proposition 2.2. Then

$$
\pi_{*} \mathcal{O}_{C}=\mathcal{O}_{\mathbf{P}^{1}} \oplus(n-2) \mathcal{O}_{\mathbf{P}^{1}}(-r-1) \oplus \mathcal{O}_{\mathbf{P}^{1}}(-2 r-2) .
$$

Proof. Since the image of $\pi$ is smooth and of dimension $1, \pi$ is flat. Then $\pi_{*} \mathcal{O}_{C}=$

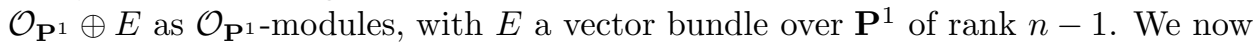
show that

$$
E=(n-2) \mathcal{O}_{\mathbf{P}^{1}}(-r-1) \oplus \mathcal{O}_{\mathbf{P}^{1}}(-2 r-2) .
$$

We have $\pi_{*} \theta=\pi_{*} \mathcal{O}_{C} \otimes \mathcal{O}_{\mathbf{P}^{1}}(r)$ and $\pi_{*} K_{C}=\pi_{*} \mathcal{O}_{C} \otimes \mathcal{O}_{\mathbf{P}^{1}}(2 r)$, by the projection formula. Any vector bundle over $\mathbf{P}^{1}$ splits; hence

$$
\pi_{*} \mathcal{O}_{C}=\mathcal{O}_{\mathbf{P}^{1}} \oplus E=\mathcal{O}_{\mathbf{P}^{1}} \oplus \mathcal{O}_{\mathbf{P}^{1}}\left(a_{1}\right) \oplus \cdots \oplus \mathcal{O}_{\mathbf{P}^{1}}\left(a_{n-1}\right)
$$

for some negative integers $a_{1}, \ldots, a_{n-1}\left(C\right.$ is connected). Then $h^{1}\left(K_{C}\right)=1$ implies that exactly one of the $a_{i}$ 's, let us say $a_{n-1}$, satisfies $a_{n-1}+2 r=-2$. On the other hand, since $\pi$ is induced by the complete linear series $|\theta|, h^{0}(\theta)=r+1=$ $h^{0}\left(\mathcal{O}_{\mathbf{P}^{1}}(r)\right)$; so $a_{i}+r \leq-1$ for all $1 \leq i \leq n-2$. Finally, since the degree of $\theta$ is $g(C)-1$, we have $h^{1}(\theta)=h^{0}(\theta)=r+1$. Since $h^{1}\left(\mathcal{O}_{\mathbf{P}^{1}}(-r-2)\right)=r+1, a_{i}+r \geq-1$ for all $1 \leq i \leq n-2$, and so $a_{i}+r=-1$ for all $1 \leq i \leq n-2$.

(2.4) Proof of Proposition 2.2. In Lemma 2.3, we have completely determined

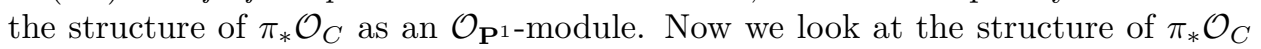


as an $\mathcal{O}_{\mathbf{P}^{1}}$-algebra. If $n=2$, it is completely determined by the branch divisor of $\pi$ on $\mathbf{P}^{1}$, since in this case $\pi$ is cyclic. If $n>2$, we observe the following:

For some $1 \leq i, j \leq n-2$, the projection of the map

$$
\mathcal{O}_{\mathbf{P}^{1}}\left(a_{i}\right) \otimes \mathcal{O}_{\mathbf{P}^{1}}\left(a_{j}\right) \longrightarrow \pi_{*} \mathcal{O}_{C} \text { to } \mathcal{O}_{\mathbf{P}^{1}}(-2 r-2)
$$

is surjective; in fact, it is an isomorphism.

This is so because otherwise $\mathcal{O}_{\mathbf{P}^{1}} \oplus \mathcal{O}_{\mathbf{P}^{1}}\left(a_{1}\right) \oplus \cdots \oplus \mathcal{O}_{\mathbf{P}^{1}}\left(a_{n-2}\right)$ would be an integral subalgebra of $\pi_{*} \mathcal{O}_{C}$, free over $\mathcal{O}_{\mathbf{P}^{1}}$ of rank $n-1$. Then $n-1$ should divide $n$, which is not possible if $n>2$.

Now we will use our knowledge of $\pi_{*} \mathcal{O}_{X}$ to study the maps $\beta(s, r)$ which appear in the statement of the proposition. We will write $\beta_{s}$ in place of $\beta(s, 1)$. Let $R_{l}=H^{0}\left(\theta^{\otimes l}\right)$. Then, since $\theta=\pi^{*} \mathcal{O}_{\mathbf{P}^{1}}(r)$, by the projection formula,

$$
\begin{gathered}
R_{1}=H^{0}\left(\mathcal{O}_{\mathbf{P}^{1}}(r)\right), \\
R_{l}=H^{0}\left(\mathcal{O}_{\mathbf{P}^{1}}(l r)\right) \oplus(n-2) H^{0}\left(\mathcal{O}_{\mathbf{P}^{1}}((l-1) r-1)\right) \oplus H^{0}\left(\mathcal{O}_{\mathbf{P}^{1}}((l-2) r-2)\right), \\
R_{l+1}=H^{0}\left(\mathcal{O}_{\mathbf{P}^{1}}((l+1) r)\right) \oplus(n-2) H^{0}\left(\mathcal{O}_{\mathbf{P}^{1}}(l r-1)\right) \oplus H^{0}\left(\mathcal{O}_{\mathbf{P}^{1}}((l-1) r-2)\right) .
\end{gathered}
$$

Therefore an element of $R_{l}$, i.e., a global section of $H^{0}\left(\theta^{\otimes l}\right)$, is a sum of $n$ components, one in each piece of the above decomposition of $R_{l}$. On the other hand, the product of an element of $R_{l}$ belonging to one of the blocks with an element of $R_{1}$ is determined by the ring structure of $\mathcal{O}_{\mathbf{P}^{1}}$ and by the module structure of $E$. More precisely, the restriction of $\beta_{l}$ to $H^{0}\left(\mathcal{O}_{\mathbf{P}^{1}}(l r)\right) \otimes H^{0}\left(\mathcal{O}_{\mathbf{P}^{1}}(r)\right)$ maps, in fact isomorphically, onto $H^{0}\left(\mathcal{O}_{\mathbf{P}^{1}}((l+1) r)\right)$. The restriction of $\beta_{l}$ to each of the blocks $H^{0}\left(\mathcal{O}_{\mathbf{P}^{1}}((l-1) r-1)\right) \otimes H^{0}\left(\mathcal{O}_{\mathbf{P}^{1}}(r)\right)$ maps to the corresponding $H^{0}\left(\mathcal{O}_{\mathbf{P}^{1}}(l r-1)\right)$. This restriction is 0 if $(l-1) r-1$ is negative and an isomorphism otherwise. Likewise, the restriction of $\beta_{l}$ to $H^{0}\left(\mathcal{O}_{\mathbf{P}^{1}}((l-2) r-2)\right) \otimes H^{0}\left(\mathcal{O}_{\mathbf{P}^{1}}(r)\right)$ goes to $H^{0}\left(\mathcal{O}_{\mathbf{P}^{1}}((l-1) r-2)\right)$, being 0 if $(l-2) r-2$ is negative and an isomorphism otherwise. Therefore it is crucial to tell which blocks of a given $R_{l}$ are 0 . We have

$$
\begin{gathered}
R_{1}=H^{0}\left(\mathcal{O}_{\mathbf{P}^{1}}(r)\right), \\
R_{2}=H^{0}\left(\mathcal{O}_{\mathbf{P}^{1}}(2 r)\right) \oplus(n-2) H^{0}\left(\mathcal{O}_{\mathbf{P}^{1}}(r-1)\right),
\end{gathered}
$$

and if $l \geq 3$,

$$
R_{l}=H^{0}\left(\mathcal{O}_{\mathbf{P}^{1}}(l r)\right) \oplus(n-2) H^{0}\left(\mathcal{O}_{\mathbf{P}^{1}}((l-1) r-1)\right) \oplus H^{0}\left(\mathcal{O}_{\mathbf{P}^{1}}((l-2) r-2)\right) .
$$

All the direct summands appearing in the above formulae are nonzero, except $H^{0}\left(\mathcal{O}_{\mathbf{P}^{1}}((l-2) r-2)\right)$ when $l=3$ and $r=1$ and $(n-2) H^{0}\left(\mathcal{O}_{\mathbf{P}^{1}}((l-1) r-1)\right)$ for all $l$ and all $r$ when $n=2$. We now determine the image of $\beta_{l}$. If $l=1$, the image of $\beta_{1}$ is $H^{0}\left(\mathcal{O}_{\mathbf{P}^{1}}(2 r)\right)$, which has codimension $(n-2) r$ in $R_{2}$. If $l=2$, the image of $\beta_{2}$ is $H^{0}\left(\mathcal{O}_{\mathbf{P}^{1}}(3 r)\right) \oplus(n-2) H^{0}\left(\mathcal{O}_{\mathbf{P}^{1}}(2 r-1)\right)$, which has codimension $r-1$ in $R_{3}$. If $l=3$ and $r \geq 2$ or if $l \geq 4$, the image of $\beta_{l}$ is all $R_{l}$, i.e., $\beta_{l}$ surjects. All this proves a.1), a.2), a.5) and b). If $r=1$, the image of $\beta(3,1)$ is $H^{0}\left(\mathcal{O}_{\mathbf{P}^{1}}(4 r)\right) \oplus(n-2) H^{0}\left(\mathcal{O}_{\mathbf{P}^{1}}(3 r-1)\right)$, which has codimension 1 in $R_{4}$. This proves a.3). If $r=1$ and $n=2$, the image of $\beta(2,2)$ is $H^{0}\left(\mathcal{O}_{\mathbf{P}^{1}}(4 r)\right)$, which has codimension 1 in $R_{4}$. This proves the first claim in a.4) and the last sentence of Proposition 2.2. Finally, if $n>2$, recall (see 2.4.1) that for some $1 \leq i, j \leq n-2$, the projection of the map

$$
\mathcal{O}_{\mathbf{P}^{1}}\left(a_{i}\right) \otimes \mathcal{O}_{\mathbf{P}^{1}}\left(a_{j}\right) \longrightarrow \pi_{*} \mathcal{O}_{C}
$$


to $\mathcal{O}_{\mathbf{P}^{1}}(-4)$ is surjective; in fact, it is an isomorphism. Then, if $n>2$, the image of $\beta(2,2)$ is all $R_{4}$. This proves the second part of a.4).

Remark 2.5. Note that $\theta^{\otimes 2}=K_{C}$. Then a proof of a.4), alternate to the one given above, can be obtained from Noether's theorem and from the base-point-free pencil trick. The way in which Noether's theorem is related to the algebra structure of $\pi_{*} \mathcal{O}_{C}$ is shown in GP4], where we will give a different, simple proof of this classical result for a general curve in $M_{g}^{1}$.

From Proposition 2.2 we obtain the following.

Corollary 2.6. Let $C$ be a smooth curve. Let $\theta$ be a base-point-free line bundle on $C$ such that $\theta^{\otimes 2}=K_{C}$. Let $\pi$ be the morphism induced by $|\theta|$, let $n$ be the degree of $\pi$ and assume that $\pi(C)$ is a rational normal curve of degree $r$. Let $R$ be $\bigoplus_{l=0}^{\infty} H^{0}\left(\theta^{\otimes l}\right)$. Then:

1) if $r=1$ and $n=2$, the ring $R$ is generated by its part of degree 1 and one generator in degree 4 ;

2) if $r=1$ and $n>2$, the ring $R$ is generated by its part of degree 1 and $n-2$ generators in degree 2 ;

3) if $r>1$, the ring $R$ is generated by its part of degree $1, r(n-2)$ generators in degree 2 and $r-1$ generators in degree 3 .

Proof. To know in what degrees we need generators, we look at the maps $\beta(s, t)$ of multiplication of sections. Precisely the number of generators needed in degree $l+1$ is the codimension in $R_{l+1}$ of the sum of the images of $\beta(l, 1)$, $\beta(l-1,2), \ldots, \beta\left(\left\lfloor\frac{l+1}{2}\right\rfloor,\left\lceil\frac{l+1}{2}\right\rceil\right)$. In particular, $R$ is generated in degree less than or equal to $l$ if $\beta_{k}$ surjects for all $k \geq l$. Thus 1) follows from part a) of Proposition 2.2 and from the fact that the images of $\beta(3,1)$ and $\beta(2,2)$ are equal. 2$)$ follows likewise from part a) of Proposition 2.2 (note that in this case $\beta(2,2)$ surjects). Finally, 3) follows from part b) of Proposition.

(2.7) Proof of Theorem 2.1. The proof rests on Proposition 2.2. The idea is "to lift" the generators of $R$ to the canonical ring of $S$. Let us define

$$
H^{0}\left(K_{S}^{\otimes s}\right) \otimes H^{0}\left(K_{S}^{\otimes r}\right) \stackrel{\alpha(s, t)}{\longrightarrow} H^{0}\left(K_{S}^{\otimes s+t}\right),
$$

and let us denote $\alpha(s, 1)$ as $\alpha_{s}$. As in the case of $R$, the images of $\alpha(s, t)$ will tell us the generators of each graded piece of the canonical ring of $S$. In fact, it will suffice to prove the following:

(a) If $r=1$ and $n=2, \alpha_{l}$ surjects for all $l \geq 1$, except if $l=3$. The images of $\alpha_{3}=\alpha(3,1)$ and $\alpha(2,2)$ are equal and have codimension 1 in $H^{0}\left(K_{S}^{\otimes 4}\right)$.

(b) If $r=1$ and $n>2, \alpha_{l}$ surjects for all $l \geq 1$, except if $l=1,3$. The image of $\alpha_{1}$ has codimension $n-2$ in $H^{0}\left(K_{S}^{\otimes 2}\right)$. The map $\alpha(2,2)$ is surjective.

(c) If $r \geq 2, \alpha_{l}$ is surjective if $l \geq 3$. The image of $\alpha_{1}$ has codimension $r(n-2)$ in $H^{0}\left(K_{S}^{\otimes 2}\right)$. The image of $\alpha_{2}$ has codimension $r-1$ in $H^{0}\left(K_{S}^{\otimes 3}\right)$.

Thus we proceed to prove (a), (b), (c). Recall that $Y=\varphi(S)$ is an irreducible variety of minimal degree and, in particular, normal. On the other hand, the locus of the points of $Y$ with non-finite fibers has codimension 2. Thus, using Bertini's theorem, we can choose a smooth curve $C$ of $\left|K_{S}\right|$ such that the restriction of the canonical morphism of $S$ to $C$ is finite (and flat) onto a smooth rational normal curve of degree $r$. Let us denote by $\theta$ the restriction of $K_{S}$ to $C$. By adjunction, 
$K_{C}=\theta^{\otimes 2}$. Since $K_{S}$ is base-point-free, so is $\theta$. Finally, since $H^{1}\left(\mathcal{O}_{X}\right)=0, \pi$ is induced by the complete linear series $|\theta|$, and therefore $C, \theta$ and $\pi$ satisfy the hypothesis of Proposition 2.2.

We prove first the statements in (a), (b) and (c) regarding the maps $\alpha_{l}$. Consider the following commutative diagram:

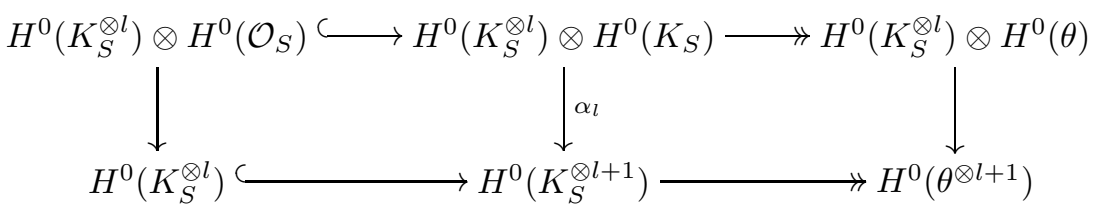

The rightmost horizontal arrows are surjective because $H^{1}\left(\mathcal{O}_{S}\right)=0$, by Serre duality and by Kawamata-Viehweg vanishing. The left vertical arrow trivially surjects. The right vertical arrow is the composition of the map $H^{0}\left(K_{S}^{\otimes l}\right) \otimes H^{0}(\theta) \longrightarrow$ $H^{0}\left(\theta^{\otimes l}\right) \otimes H^{0}(\theta)$, which is surjective for all $l \geq 1$ again because $H^{1}\left(\mathcal{O}_{S}\right)=0$, by Serre duality and by Kawamata-Viehweg vanishing, and the map $\beta_{l}$ of multiplication of global sections on $C$, studied in Proposition 2.2. Then it follows from chasing the diagram that the map $H^{0}\left(K_{S}^{\otimes l+1}\right) \longrightarrow H^{0}\left(\theta^{\otimes l+1}\right)$ maps the image of $\alpha_{l}$ onto the image of $\beta_{l}$, and that the codimension of the image of $\beta_{l}$ in $H^{0}\left(\theta^{\otimes l+1}\right)$ is equal to the codimension of the image of $\alpha_{l}$ in $H^{0}\left(K_{S}^{\otimes l+1}\right)$. This, together with Proposition 2.2, a.1, a.2, a.3, a.5 and b, proves the claims in (a), (b) and (c) concerning the codimensions of the images of the maps $\alpha_{l}$.

Thus the only things left to prove are the claims about $\alpha(2,2)$ when $r=1$. We consider the commutative diagram

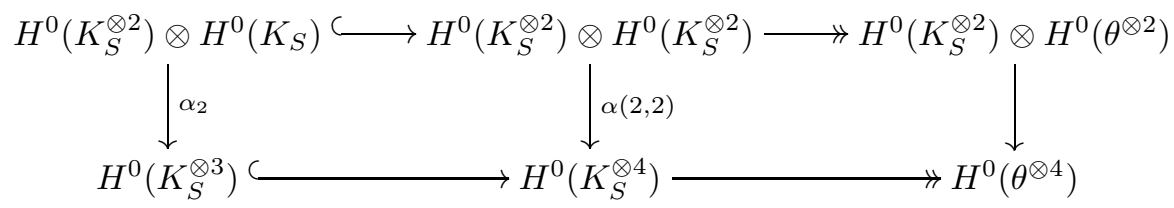

The rightmost horizontal arrows are surjective because $H^{1}\left(\mathcal{O}_{S}\right)=0$ and by Serre duality, and by Kawamata-Viehweg vanishing. The left vertical arrow surjects, as we have already proven. The right vertical arrow is the composition of the map $H^{0}\left(K_{S}^{\otimes 2}\right) \otimes H^{0}\left(\theta^{\otimes 2}\right) \longrightarrow H^{0}\left(\theta^{\otimes 2}\right) \otimes H^{0}\left(\theta^{\otimes 2}\right)$, which is surjective because $S$ is regular and by Serre duality, and the map $\beta(2,2)$ of multiplication of global sections on $C$. Then it follows from chasing the diagram that the map $H^{0}\left(K_{S}^{\otimes 4}\right) \rightarrow H^{0}\left(\theta^{\otimes 4}\right)$ maps the image of $\alpha(2,2)$ onto the image of $\beta(2,2)$, and that the codimension of the image of $\beta(2,2)$ in $H^{0}\left(\theta^{\otimes 4}\right)$ is equal to the codimension of the image of $\alpha(2,2)$ in $H^{0}\left(K_{S}^{\otimes 4}\right)$. On the other hand, we know that the image of $\beta(2,2)$ and the image of $\beta_{3}=\beta(3,1)$ are equal of codimension 1 in $H^{0}\left(\theta^{\otimes 4}\right)$, if $r=1$ and $n=2$. Thus we conclude that the images of $\alpha(3,1)$ and $\alpha(2,2)$ in $H^{0}\left(K_{S}^{\otimes 4}\right)$ are also equal and of codimension 1. Finally, if $r=1$ and $n>2, \beta(2,2)$ surjects by Proposition 2.2.a.4. Thus we conclude that if $r=1$ and $n>2$, then $\alpha(2,2)$ surjects.

Theorem 2.1 complements known results on generation of the canonical ring of smooth, regular surfaces of general type. Ciliberto and Green (cf. G], Theorem 3.9 .3 , and [Ci]) proved that, given a smooth surface of general type with $h^{1}\left(\mathcal{O}_{S}\right)=$ 0 and $K_{S}$ globally generated and $\varphi$ being the canonical morphism, a sufficient condition for the canonical ring of $S$ to be generated in degree less than or equal to 2 is that: 
(1) $\varphi$ does not map $S$ generically $2: 1$ onto $\mathbf{P}^{2}$, and

(2) $\varphi(S)$ is not a surface of minimal degree other than linear $\mathbf{P}^{2}$.

As a corollary of the Ciliberto and Green result and of Theorem 2.1, we obtain the following:

Corollary 2.8. Let $S$ be a smooth regular surface of general type and such that $K_{S}$ is globally generated. Let $\varphi$ be the canonical morphism of $S$. The canonical ring of $S$ is generated in degree less than or equal to 2 if and only if

(1) $\varphi$ does not map $S$ generically $2: 1$ onto $\mathbf{P}^{2}$, and

(2) $\varphi(S)$ is not a surface of minimal degree other than linear $\mathbf{P}^{2}$.

\section{EXAMPles OF SURFACES OF GENERAL TYPE}

In this section we construct some new examples of surfaces of general type that satisfy the hypothesis of Theorem 2.1. The easiest way one could think of producing examples would be to build suitable cyclic covers of surfaces of minimal degree. However, as the next proposition shows, only low degree cyclic covers can be induced by the canonical morphism of a regular surface. So we have to employ other means to construct these examples.

Proposition 3.1. Let $X$ be a surface of general type with at worst canonical singularities and with base-point-free canonical bundle. Assume that the complete canonical series of $X$ restricts to a complete linear series on a general member of the canonical series (e.g., if $X$ is regular). Let $\varphi: X \longrightarrow Y$ be the canonical morphism to a surface of minimal degree. Let $n$ be the degree of $\varphi$. Let $U$ be a smooth open set of $Y$ whose complement has codimension 2 and let $L$ be a line bundle on $U$. Assume that

$$
\left.\left(\varphi_{*} \mathcal{O}_{X}\right)\right|_{U}=\mathcal{O}_{U} \oplus L^{-1} \oplus \cdots \oplus L^{\otimes 1-n} .
$$

Then $n=2$ or 3 .

Proof. Let $H$ be a general hyperplane section of $Y$ contained in $U$ and let $C$ be the inverse image of $H$ by $\varphi$. Then $C$ is a smooth irreducible member of $\left|K_{X}\right|$ and $H$ is a smooth rational normal curve. By assumption the morphism $\left.\varphi\right|_{C}: C \longrightarrow H$ is induced by the complete linear series of a line bundle $\theta$. By adjunction $\theta^{\otimes 2}=K_{C}$. Thus $C, \theta$ and $\left.\varphi\right|_{C}$ satisfy the hypothesis of Lemma 2.3, and

$$
\left(\left.\varphi\right|_{C}\right)_{*} \mathcal{O}_{C}=\mathcal{O}_{\mathbf{P}^{1}} \oplus(n-2) \mathcal{O}_{\mathbf{P}^{1}}(-r-1) \oplus \mathcal{O}_{\mathbf{P}^{1}}(-2 r-2) .
$$

On the other hand, $\left(\left.\varphi\right|_{C}\right)_{*} \mathcal{O}_{C}$ is equal to the restriction of $\varphi_{*} \mathcal{O}_{X}$ to $H$, and hence

$$
\left(\left.\varphi\right|_{C}\right)_{*} \mathcal{O}_{C}=\mathcal{O}_{\mathbf{P}^{1}} \oplus L^{\prime-1} \oplus \cdots \oplus L^{\prime 1-n}
$$

where $L^{\prime}$ is the restriction of $L$ to $H$. The only way in which $\left(\left.\varphi\right|_{C}\right)_{*} \mathcal{O}_{C}$ can have these two splittings is when $n=2$ or 3 .

Corollary 3.2. Let $X$ be a regular surface of general type with at worst canonical singularities and with base-point-free canonical bundle. Let $Y$ be the image of $X$ by its canonical morphism $X \stackrel{\varphi}{\rightarrow} Y$. If $Y$ is a surface of minimal degree and $\varphi$ is a cyclic cover, then the degree of $\varphi$ is 2 or 3 .

The next proposition also rules out many possible examples of covers of odd degree: 
Proposition 3.3. Let $X$ be a surface of general type with at worst canonical singularities whose canonical divisor is base-point-free. Let $\varphi$ be a morphism induced by a subseries of $\left|K_{X}\right|$. If $\varphi$ is generically finite onto a smooth scroll $Y \subset \mathbf{P}^{N}$, then the degree of $\varphi$ is even. In particular, there are not generically finite covers of odd degree of smooth rational normal scrolls induced by subseries of $K_{X}$.

Proof. Let $f$ be a fiber of $Y$ and let $C$ be a section of $Y$. Let $-d=C^{2}$. Since $Y$ is a scroll, its hyperplane section is linearly equivalent to $C+m f$, for some integer $m$. Then $K_{X}=\varphi^{*}(C+m f)$. Then $\operatorname{deg} \varphi=\left(\varphi^{*} f\right) \cdot\left(\varphi^{*} C\right)=\left(\varphi^{*} f\right) \cdot\left(K_{X}-m \varphi^{*} f\right)=$ $\left(\varphi^{*} f\right) \cdot\left(K_{X}+\varphi^{*} f\right)$, which is an even number.

Now we construct some examples of regular minimal surfaces $X$ whose canonical morphism $\varphi$ maps onto a variety of minimal degree, and also mention known ones relevant to this paper.

The cases when $\varphi$ is a generically finite morphism and has degree 2 or 3 have been completely studied by Horikawa and Konno (see [H1], Theorem 1.6, [H2], Theorem 2.3.I, [H3], Theorem 4.1 and [Kon, Lemma 2.2 and Theorem 2.3; see also Mendes Lopes and Pardini, [MP]). As it turns out, there exist generically double covers of linear $\mathbf{P}^{2}$, the Veronese surface, smooth rational normal scrolls $S(a, b)$ with $b \leq 4$, and cones over rational normal curves of degree 2,3 and 4 and generically triple covers of $\mathbf{P}^{2}$ (in particular, cyclic triple covers of $\mathbf{P}^{2}$ ramified along a sextic with suitable singularities) and of the cones over rational normal curves of degree 2 and 3. Horikawa (see [H4], Theorem 2.1) also describes all generically finite quadruple covers $X \stackrel{\varphi}{\longrightarrow} Y$, where $X$ is a smooth, minimal regular surface, $\varphi$ is the canonical morphism of $X$, and $Y$ is linear $\mathbf{P}^{2}$.

The examples of Horikawa and Konno just reviewed are examples of covers of degree less than or equal to 3 of surfaces of minimal degree and quadruple covers of $\mathbf{P}^{2}$. We now construct three new sets of examples of regular surfaces of general type that are quadruple covers of surfaces of minimal degree under the canonical morphism. These examples are 4:1 covers of smooth rational normal scrolls isomorphic to the Hirzebruch surfaces $\mathbf{F}_{0}$ and $\mathbf{F}_{1}$, and of quadric cones in $\mathbf{P}^{3}$.

Example 3.4. We construct finite quadruple covers $X \stackrel{\varphi}{\rightarrow} Y$, where $X$ is a smooth minimal regular surface of general type, $\varphi$ is the canonical morphism of $X$, and $Y$ is a smooth rational scroll $S(m, m), m \geq 1$.

Let $f$ be a fiber of one of the fibrations of $\mathbf{P}^{1}$ and let $f^{\prime}$ be a fiber of the other fibration. Then $Y$ is $\mathbf{P}^{1} \times \mathbf{P}^{1}$, and it is embedded in $\mathbf{P}^{2 m+1}$ by $\left|f+m f^{\prime}\right|$ or by $\left|f^{\prime}+m f\right|$. If $Y$ is embedded by $\left|f+m f^{\prime}\right|$, let $a_{1}, a_{2}, b_{1}$ and $b_{2}$ satisfy the following:

$$
\begin{gathered}
\text { either } a_{1}=1, a_{2}=2, b_{1}=m+1 \text { and } b_{2}=1, \\
\quad \text { or } a_{1}=2, a_{2}=1, b_{1}=1 \text { and } b_{2}=m+1 .
\end{gathered}
$$

If $Y$ is embedded by $\left|f^{\prime}+m f\right|$, let $a_{1}, a_{2}, b_{1}$ and $b_{2}$ satisfy the following:

$$
\begin{gathered}
\text { either } b_{1}=1, b_{2}=2, a_{1}=m+1 \text { and } a_{2}=1, \\
\text { or } b_{1}=2, b_{2}=1, a_{1}=1 \text { and } a_{2}=m+1 .
\end{gathered}
$$

For $i=1,2$, let $D_{i}$ be a smooth divisor linearly equivalent to $2\left(a_{i} f+b_{i} f^{\prime}\right)$ such that $D_{1}$ and $D_{2}$ intersect at $D_{1} \cdot D_{2}$ distinct points. Those divisors exist because by the choices of $a_{1}, a_{2}, b_{1}$ and $b_{2}$, both $2\left(a_{1} f+b_{1} f^{\prime}\right)$ and $2\left(a_{2} f+b_{2} f^{\prime}\right)$ are very ample. Let $X^{\prime} \stackrel{\varphi_{1}}{\longrightarrow} Y$ be the double cover of $Y$ ramified along $D_{1}$. Since $D_{1}$ is 
smooth, so is $X^{\prime}$. Let $D_{2}^{\prime}$ be the inverse image in $X^{\prime}$ of $D_{2}$ by $\varphi_{1}$. Since $D_{2}$ is smooth and meets $D_{1}$ at distinct points, $D_{2}^{\prime}$ is also smooth. Let $X \stackrel{\varphi_{2}}{\longrightarrow} X^{\prime}$ be the double cover of $X^{\prime}$ ramified along $D_{2}^{\prime}$. Since $X^{\prime}$ and $D_{2}^{\prime}$ are both smooth, so is $X$. Let $\varphi=\varphi_{1} \circ \varphi_{2}$. Now we will show that $X$ is a regular surface of general type, that $K_{X}=\varphi^{*} \mathcal{O}_{Y}(1)$, and that $\varphi$ is induced by the complete canonical series of $X$. First we find out the structure of $\varphi_{*} \mathcal{O}_{X}$ as a module over $\mathcal{O}_{Y}$. Recall that $\varphi_{2 *} \mathcal{O}_{X}=\mathcal{O}_{X^{\prime}} \oplus \varphi_{1}{ }^{*} \mathcal{O}_{Y}\left(-a_{2} f-b_{2} f^{\prime}\right)$. Then

$$
\varphi_{*} \mathcal{O}_{X}=\varphi_{1_{*}} \mathcal{O}_{X^{\prime}} \oplus \varphi_{1_{*}}\left(\varphi_{1}{ }^{*} \mathcal{O}_{Y}\left(-a_{2} f-b_{2} f^{\prime}\right)\right)
$$

Since $\varphi_{1 *} \mathcal{O}_{X^{\prime}}=\mathcal{O}_{Y} \oplus \mathcal{O}_{Y}\left(-a_{1} f-b_{1} f^{\prime}\right)$, then by the projection formula we have $\varphi_{*} \mathcal{O}_{X}=\mathcal{O}_{Y} \oplus \mathcal{O}_{Y}\left(-a_{1} f-b_{1} f^{\prime}\right) \oplus \mathcal{O}_{Y}\left(-a_{2} f-b_{2} f^{\prime}\right) \oplus \mathcal{O}_{Y}\left(-\left(a_{1}+a_{2}\right) f-\left(b_{1}+b_{2}\right) f^{\prime}\right)$. We see now that $X$ is regular. Recall that $H^{1}\left(\mathcal{O}_{X}\right)=H^{1}\left(\varphi_{*} \mathcal{O}_{X}\right)$. Our choice of $a_{1}, a_{2}, b_{1}$ and $b_{2}$ implies that $a_{1} f+b_{1} f^{\prime}$ and $a_{2} f+b_{2} f^{\prime}$ are both very ample; thus, by Kodaira vanishing,

$$
\begin{gathered}
H^{1}\left(\mathcal{O}_{Y}\left(-a_{1} f-b_{1} f^{\prime}\right)\right)=H^{1}\left(\mathcal{O}_{Y}\left(-a_{2} f-b_{2} f^{\prime}\right)\right) \\
=H^{1}\left(\mathcal{O}_{Y}\left(-\left(a_{1}+a_{2}\right) f-\left(b_{1}+b_{2}\right) f^{\prime}\right)\right)=0 .
\end{gathered}
$$

Then, since $H^{1}\left(\mathcal{O}_{Y}\right)$ also vanishes, so do $H^{1}\left(\varphi_{*} \mathcal{O}_{X}\right)$ and $H^{1}\left(\mathcal{O}_{X}\right)$. We now compute $K_{X}$. Since $\varphi_{2}$ is a double cover ramified along $D_{2}^{\prime}$,

$$
K_{X}=\varphi_{2}{ }^{*}\left(K_{X^{\prime}} \otimes \varphi_{1}^{*}\left(\mathcal{O}_{Y}\left(a_{2} f+b_{2} f^{\prime}\right)\right) .\right.
$$

For a similar reason,

$$
K_{X^{\prime}}=\varphi_{1}^{*}\left(K_{Y} \otimes \mathcal{O}_{Y}\left(a_{1} f+b_{1} f^{\prime}\right)\right)
$$

Then

$$
K_{X}=\varphi^{*}\left(K_{Y} \otimes \mathcal{O}_{Y}\left(\left(a_{1}+a_{2}\right) f+\left(b_{1}+b_{2}\right) f^{\prime}\right)\right) .
$$

Since $K_{Y}=\mathcal{O}_{Y}\left(-2 f-2 f^{\prime}\right)$, it follows again from the choices of $a_{1}, a_{2}, b_{1}$ and $b_{2}$ that $K_{X}=\varphi^{*} \mathcal{O}_{Y}(1)$. Finally, to see that $\varphi$ is induced by the complete canonical linear series of $X$, we compute $H^{0}\left(K_{X}\right)$. We do the computation in the case $\mathcal{O}_{Y}(1)=\mathcal{O}_{Y}\left(f+m f^{\prime}\right)$. The case $\mathcal{O}_{Y}(1)=\mathcal{O}_{Y}\left(m f+f^{\prime}\right)$ is analogous. Since $K_{X}=\varphi^{*} \mathcal{O}_{Y}(1)$,

$$
\begin{gathered}
H^{0}\left(K_{X}\right)=H^{0}\left(\mathcal{O}_{Y}(1)\right) \oplus H^{0}\left(\mathcal{O}_{Y}\left(\left(1-a_{1}\right) f+\left(m-b_{1}\right) f^{\prime}\right)\right) \\
\oplus H^{0}\left(\mathcal{O}_{Y}\left(\left(1-a_{2}\right) f+\left(m-b_{2}\right) f^{\prime}\right)\right) \\
\oplus H^{0}\left(\mathcal{O}_{Y}\left(\left(1-a_{1}-a_{2}\right) f+\left(m-b_{1}-b_{2}\right) f^{\prime}\right)\right)
\end{gathered}
$$

Again, by the choices of $a_{1}, a_{2}, b_{1}$ and $b_{2}$, the last three direct sums of the above expression are 0 . So $\varphi$ is indeed induced by the complete canonical series of $X$.

Example 3.5. We construct finite quadruple covers $X \stackrel{\varphi}{\rightarrow} Y$, where $X$ is a smooth regular surface of general type with base-point-free canonical bundle, $\varphi$ is the canonical morphism of $X$, and $Y$ is a smooth rational scroll $S(m-1, m), m \geq 2$.

Let $C_{0}$ be the minimal section of $\mathbf{F}_{1}$ and let $f$ be one of the fibers. Then $Y$ is $\mathbf{F}_{1}$, and it is embedded in $\mathbf{P}^{2 m}$ by $\left|C_{0}+m f\right|$. Let $a_{1}, a_{2}, b_{1}$ and $b_{2}$ satisfy the following:

$$
\begin{gathered}
\text { either } a_{1}=1, a_{2}=2, b_{1}=m+1 \text { and } b_{2}=2, \\
\quad \text { or } a_{1}=2, a_{2}=1, b_{1}=2 \text { and } b_{2}=m+1 .
\end{gathered}
$$

For $i=1,2$, let $D_{i}$ be a smooth divisor linearly equivalent to $2\left(a_{i} C_{0}+b_{i} f\right)$, such that $D_{1}$ and $D_{2}$ intersect at $D_{1} \cdot D_{2}$ distinct points. The fact that such divisors exist 
follows from our choice of $a_{1}, a_{2}, b_{1}$ and $b_{2}$, which implies that of the linear systems of $D_{1}$ and $D_{2}$, one is very ample, and the other is base-point-free. Let $X^{\prime} \stackrel{\varphi_{1}}{\longrightarrow} Y$ be the double cover of $Y$ ramified along $D_{1}$. Since $D_{1}$ is smooth, so is $X^{\prime}$. Let $D_{2}^{\prime}$ be the inverse image in $X^{\prime}$ of $D_{2}$ by $\varphi_{1}$. Since $D_{2}$ is smooth and meets $D_{1}$ transversally, $D_{2}^{\prime}$ is also smooth. Let $X \stackrel{\varphi_{2}}{\longrightarrow} X^{\prime}$ be the double cover of $X^{\prime}$ ramified along $D_{2}^{\prime}$. Since $X^{\prime}$ and $D_{2}^{\prime}$ are both smooth, so is $X$. Let $\varphi=\varphi_{1} \circ \varphi_{2}$. Now we will show that $X$ is a regular surface of general type, that $K_{X}=\varphi^{*} \mathcal{O}_{Y}(1)$, and that $\varphi$ is induced by the complete canonical series of $X$. First we find the structure of $\varphi_{*} \mathcal{O}_{X}$ as a module over $\mathcal{O}_{Y}$. Recall that $\varphi_{2 *} \mathcal{O}_{X}=\mathcal{O}_{X^{\prime}} \oplus \varphi_{1}{ }^{*} \mathcal{O}_{Y}\left(-a_{2} C_{0}-b_{2} f\right)$. Then

$$
\varphi_{*} \mathcal{O}_{X}=\varphi_{1 *} \mathcal{O}_{X^{\prime}} \oplus \varphi_{1 *}\left(\varphi_{1}^{*} \mathcal{O}_{Y}\left(-a_{2} C_{0}-b_{2} f\right)\right)
$$

Since $\varphi_{1 *} \mathcal{O}_{X^{\prime}}=\mathcal{O}_{Y} \oplus \mathcal{O}_{Y}\left(-a_{1} C_{0}-b_{1} f\right)$, then by the projection formula we have

$$
\begin{aligned}
\varphi_{*} \mathcal{O}_{X}= & \mathcal{O}_{Y} \oplus \mathcal{O}_{Y}\left(-a_{1} C_{0}-b_{1} f\right) \oplus \mathcal{O}_{Y}\left(-a_{2} C_{0}-b_{2} f\right) \\
& \oplus \mathcal{O}_{Y}\left(-\left(a_{1}+a_{2}\right) C_{0}-\left(b_{1}+b_{2}\right) f\right) .
\end{aligned}
$$

We see now that $X$ is regular. Recall that $H^{1}\left(\mathcal{O}_{X}\right)=H^{1}\left(\varphi_{*} \mathcal{O}_{X}\right)$. Our choices of $a_{1}, a_{2}, b_{1}$ and $b_{2}$ imply that $a_{1} C_{0}+b_{1} f$ and $a_{2} C_{0}+b_{2} f$ are both base-point-free and big divisors; thus, by Kawamata-Viehweg vanishing,

$$
\begin{gathered}
H^{1}\left(\mathcal{O}_{Y}\left(-a_{1} C_{0}-b_{1} f\right)\right)=H^{1}\left(\mathcal{O}_{Y}\left(-a_{2} C_{0}-b_{2} f\right)\right) \\
=H^{1}\left(\mathcal{O}_{Y}\left(-\left(a_{1}+a_{2}\right) C_{0}-\left(b_{1}+b_{2}\right) f\right)\right)=0 .
\end{gathered}
$$

Then, since $H^{1}\left(\mathcal{O}_{Y}\right)$ also vanishes, so does $H^{1}\left(\varphi_{*} \mathcal{O}_{X}\right)$ and therefore $H^{1}\left(\mathcal{O}_{X}\right)$. We now compute $K_{X}$. Since $\varphi_{2}$ is a double cover ramified along $D_{2}^{\prime}$,

$$
K_{X}=\varphi_{2}^{*}\left(K_{X^{\prime}} \otimes \varphi_{1}^{*}\left(\mathcal{O}_{Y}\left(a_{2} C_{0}+b_{2} f\right)\right) .\right.
$$

For a similar reason,

$$
K_{X^{\prime}}=\varphi_{1}^{*}\left(K_{Y} \otimes \mathcal{O}_{Y}\left(a_{1} C_{0}+b_{1} f\right)\right)
$$

Then

$$
K_{X}=\varphi^{*}\left(K_{Y} \otimes \mathcal{O}_{Y}\left(\left(a_{1}+a_{2}\right) C_{0}+\left(b_{1}+b_{2}\right) f\right)\right) .
$$

Since $K_{Y}=\mathcal{O}_{Y}\left(-2 C_{0}-3 f\right)$, it follows from our choice of $a_{1}, a_{2}, b_{1}$ and $b_{2}$ that $K_{X}=\varphi^{*} \mathcal{O}_{Y}(1)$. Finally, to see that $\varphi$ is induced by the complete canonical linear series of $X$, we compute $H^{0}\left(K_{X}\right)$. Since $K_{X}=\varphi^{*} \mathcal{O}_{Y}(1)$,

$$
\begin{gathered}
H^{0}\left(K_{X}\right)=H^{0}\left(\mathcal{O}_{Y}(1)\right) \oplus H^{0}\left(\mathcal{O}_{Y}\left(\left(1-a_{1}\right) C_{0}+\left(m-b_{1}\right) f\right)\right) \\
\oplus H^{0}\left(\mathcal{O}_{Y}\left(\left(1-a_{2}\right) C_{0}+\left(m-b_{2}\right) f\right)\right) \\
\oplus H^{0}\left(\mathcal{O}_{Y}\left(\left(1-a_{1}-a_{2}\right) C_{0}+\left(m-b_{1}-b_{2}\right) f\right)\right)
\end{gathered}
$$

Again, by the choices of $a_{1}, a_{2}, b_{1}$ and $b_{2}$, the last three direct sums of the above expression are 0 . So $\varphi$ is indeed induced by the complete canonical series of $K_{X}$.

Remark 3.6. With the same arguments, if one allows certain mild singularities in $D_{1}$ and $D_{2}^{\prime}$, then one can construct examples of covers of $\mathbf{F}_{0}$ and $\mathbf{F}_{1}$ with at worst canonical singularities.

Finally, we construct an example of a quadruple cover of a singular surface of minimal degree. 
Example 3.7. We construct an example of a smooth, generically finite, quadruple cover $X \stackrel{\varphi}{\rightarrow} Z$ of the quadric cone $Z$ in $\mathbf{P}^{3}$, where $X$ is a regular surface of general type whose canonical divisor is base-point-free, and $\varphi$ is its canonical morphism.

Let $Y=\mathbf{F}_{2}$. Let $C_{0}$ be the minimal section of $Y$ and let $f$ be a fiber of $Y$. Let $D_{1}$ be a smooth divisor on $Y$, linearly equivalent to $2 C_{0}+6 f$ and meeting $C_{0}$ transversally. Let $D_{2}$ be a smooth divisor on $Y$, linearly equivalent to $3 C_{0}+6 f$ and meeting $D_{1}$ transversally. Such divisors $D_{1}$ and $D_{2}$ exist, because $2 C_{0}+6 f$ is very ample and $3 C_{0}+6 f$ is base-point-free. Note also that, since $\left(3 C_{0}+6 f\right) \cdot C_{0}=0$, $C_{0}$ and $D_{2}$ do not meet. Let $X^{\prime} \stackrel{\varphi_{1}}{\longrightarrow} Y$ be the double cover of $Y$ along $D_{1}$. Since $D_{1}$ is smooth, so is $X^{\prime}$. Since $D_{1}$ meets $C_{0}$ at two distinct points, the pullback $C_{0}^{\prime}$ of $C_{0}$ by $\varphi_{1}$ is a smooth line with self-intersection -4 . Let $D_{2}^{\prime}$ be the pullback of $D_{2}$ by $\varphi_{1}$. Since $D_{1}$ and $D_{2}$ meet transversally, $D_{2}^{\prime}$ is smooth, and since $D_{2}$ and $C_{0}$ do not meet, neither do $D_{2}^{\prime}$ and $C_{0}^{\prime}$. Let $L_{2}^{\prime}$ be the pullback of $2 C_{0}+3 f$ by $\varphi_{1}$. Let $X \stackrel{\varphi_{2}}{\longrightarrow} X^{\prime}$ be the double cover of $X^{\prime}$ along $D_{2}^{\prime} \cup C_{0}^{\prime}$. Since $D_{2}^{\prime} \cup C_{0}^{\prime}$ is smooth, so is $X$. Let $\varphi=\varphi_{1} \circ \varphi_{2}$. Then

$$
\begin{gathered}
\varphi_{*} \mathcal{O}_{X}=\varphi_{1 *} \varphi_{2 *} \mathcal{O}_{X}=\varphi_{1 *}\left(\mathcal{O}_{X^{\prime}} \oplus L_{2}^{\prime *}\right) \\
=\mathcal{O}_{Y} \oplus \mathcal{O}_{Y}\left(-C_{0}-3 f\right) \oplus \mathcal{O}_{Y}\left(-2 C_{0}-3 f\right) \oplus \mathcal{O}_{Y}\left(-3 C_{0}-6 f\right) .
\end{gathered}
$$

Since $C_{0}+3 f$ and $3 C_{0}+6 f$ are big and base-point-free, by Kawamata-Viehweg vanishing and Serre duality, $H^{1}\left(\mathcal{O}_{Y}\left(-C_{0}-3 f\right)\right)=H^{1}\left(\mathcal{O}_{Y}\left(-3 C_{0}-6 f\right)\right)=0$. By Serre duality, $H^{1}\left(\mathcal{O}_{Y}\left(-2 C_{0}-3 f\right)\right)=H^{1}\left(\mathcal{O}_{Y}(-f)\right)^{*}=0$. Then, since $H^{1}\left(\mathcal{O}_{Y}\right)=0$, $X$ is regular. Arguing as in Examples 3.4 and 3.5, we see that

$$
K_{X}=\varphi^{*}\left(K_{Y} \otimes \mathcal{O}_{Y}\left(3 C_{0}+6 f\right)\right)=\varphi^{*} \mathcal{O}_{Y}\left(C_{0}+2 f\right) .
$$

Now we compute $H^{0}\left(K_{X}\right)$. Using the projection formula and (3.7.1) and (3.7.2), we obtain that

$$
\begin{aligned}
H^{0}\left(K_{X}\right)= & H^{0}\left(\mathcal{O}_{Y}\left(C_{0}+2 f\right)\right) \oplus H^{0}\left(\mathcal{O}_{Y}(-f)\right) \oplus H^{0}\left(\mathcal{O}_{Y}\left(-C_{0}-f\right)\right) \\
& \oplus H^{0}\left(\mathcal{O}_{Y}\left(-2 C_{0}-4 f\right)\right)=H^{0}\left(\mathcal{O}_{Y}\left(C_{0}+2 f\right)\right)
\end{aligned}
$$

Thus the canonical morphism of $X$ is the composition of $\varphi$ and the morphism $Y \stackrel{\phi}{\rightarrow} Z \subset \mathbf{P}^{3}$, induced by the complete linear series of $C_{0}+2 f$. Since $\phi$ contracts $C_{0}$, the canonical morphism of $X$ is not finite, but it is generically finite of degree 4 onto $Z$, which is a surface of minimal degree, as we wanted.

On the other hand, if $C_{0}^{\prime \prime}$ is the pullback of $C_{0}$ by $\varphi$, then $C_{0}^{\prime \prime}$ is a smooth line with self-intersection -2 . Thus the morphism $\phi \circ \varphi$ also factors as $\varphi^{\prime} \circ \psi$, where

$$
X \stackrel{\psi}{\longrightarrow} \bar{X}
$$

is the morphism from $X$ to its canonical model $\bar{X}$ and

$$
\bar{X} \stackrel{\varphi^{\prime}}{\longrightarrow} Z
$$

is the canonical morphism of $\bar{X}$. Thus $\varphi^{\prime}$ is an example of a finite, $4: 1$ canonical morphism from a regular surface of general type with canonical singularities onto a singular surface of minimal degree. 


\section{Applichtions to Calabi-Yau threefolds}

The results proved in Sections 2 and 3 have ramifications for Calabi-Yau threefolds. Recall that if $X$ is a Calabi-Yau threefold and $B$ is a big and base-point-free divisor, a general member of $|B|$ is a surface of general type. Then the geometry and properties of surfaces of general type are directly related to those of Calabi-Yau threefolds. Concretely, the results we have obtained in Section 2 on the canonical ring of surfaces of general type can be "lifted" to achieve analogous results for Calabi-Yau threefolds in a way similar to the way in which our study of rings of curves allowed us to obtain results for surfaces of general type. On the other hand, constructing examples of Calabi-Yau threefolds has attracted the attention of geometers in recent years. One of the important sources for these examples is precisely to take covers of varieties of minimal degree. Proposition 3.1, Corollary 3.2 and Proposition 3.3 tell us features of generically finite covers of surfaces of minimal degree induced by the canonical morphism. We will see how these features pass on to generically finite morphisms from Calabi-Yau threefolds to threefolds of minimal degree, and, as a consequence, we will obtain, among other things, that many a priori possible examples of Calabi-Yau threefolds cannot exist.

We start with the Calabi-Yau threefold analog of Theorem 2.1:

Theorem 4.1. Let $X$ be a Calabi-Yau threefold with at worst canonical singularities, and let $B$ be a big and base-point-free line bundle on $X$. Let $\varphi$ be the morphism induced by $|B|$. Let $n$ be the degree of $\varphi$, and assume that the image of $\varphi$ is a variety of minimal degree $r$. Then :

1) If $n=2$ and $r=1$ (i.e., if $\varphi$ is generically $2: 1$ onto $\mathbf{P}^{3}$ ), the canonical ring of $X$ is generated by its part of degree 1 and one generator in degree 4 .

2) If $n \neq 2$ or $r \neq 1$, the canonical ring of $X$ is generated by its part of degree 1 , $r(n-2)$ generators in degree 2 and $r-1$ generators in degree 3.

Sketch of proof. The proof follows the same lines as the proof of Theorem 2.1. Let us define

$$
H^{0}\left(B^{\otimes s}\right) \otimes H^{0}\left(B^{\otimes r}\right) \stackrel{\gamma(s, t)}{\longrightarrow} H^{0}\left(B^{\otimes s+t}\right),
$$

and denote $\gamma(s, 1)$ as $\gamma_{s}$. The images of $\gamma(s, t)$ will tell us the generators of each graded piece of the ring $\bigoplus_{l=0}^{\infty} H^{0}\left(B^{\otimes l}\right)$. In fact, it would suffice to prove the following:

(a) If $r=1$ and $n=2, \gamma_{l}$ surjects for all $l \geq 1$, except if $l=3$. The images of $\gamma_{3}=\gamma(3,1)$ and $\gamma(2,2)$ are equal and have codimension 1 in $H^{0}\left(B^{\otimes 4}\right)$.

(b) If $r=1$ and $n>2, \gamma_{l}$ surjects for all $l \geq 1$, except if $l=1,3$. The image of $\gamma_{1}$ has codimension $n-2$ in $H^{0}\left(B^{\otimes 2}\right)$. The map $\gamma(2,2)$ is surjective.

(c) If $r \geq 2, \gamma_{l}$ is surjective if $l \geq 3$. The image of $\gamma_{1}$ has codimension $r(n-2)$ in $H^{0}\left(B^{\otimes 2}\right)$. The image of $\gamma_{2}$ has codimension $r-1$ in $H^{0}\left(B^{\otimes 3}\right)$.

Now a suitable hyperplane section of the image of $\varphi$ is an irreducible surface $Y$ of minimal degree. Its pullback by $\varphi$ to $X$ is a surface $S$ of general type with at worst canonical singularities. Moreover, by adjunction $B \otimes \mathcal{O}_{S}=K_{S}$, and the complete linear series of $B$ restricts to the complete canonical linear series of $S$; so $\left.\varphi\right|_{S}$ is the canonical morphism of $S$. Therefore $S$ is under the hypothesis of Theorem 2.1, and the proof follows verbatim the steps given in 2.7, the role of $S$ there being played by $X$ here, the role of $C$ there being played by $S$ here and the role of Proposition 2.2 there being played by Theorem 2.1 here. 
To show what we mean, we outline how to find the images of the maps $\gamma_{l}$. We consider the following commutative diagram:

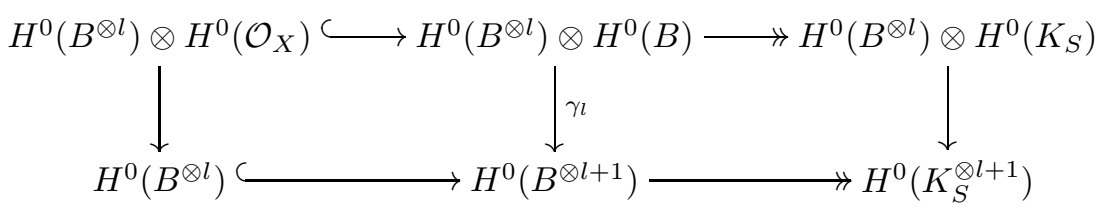

The rightmost horizontal arrows are surjective because $H^{1}\left(\mathcal{O}_{X}\right)=0$, by Serre duality and by Kawamata-Viehweg vanishing. The left vertical arrow trivially surjects. The right vertical arrow is the composition of the map $H^{0}\left(B^{\otimes l}\right) \otimes H^{0}\left(K_{S}\right) \longrightarrow$ $H^{0}\left(K_{S}^{\otimes l}\right) \otimes H^{0}\left(K_{S}\right)$, which is surjective for all $l \geq 1$ again because $H^{1}\left(\mathcal{O}_{X}\right)=0$, by Serre duality and by Kawamata-Viehweg vanishing, and the map $\alpha_{l}$ of multiplication of global sections on $S$, studied in (2.7). Then it follows from chasing the diagram that the map $H^{0}\left(B^{\otimes l+1}\right) \longrightarrow H^{0}\left(K_{S}^{\otimes l+1}\right)$ maps the image of $\gamma_{l}$ onto the image of $\alpha_{l}$ and that the codimension of the image of $\alpha_{l}$ in $H^{0}\left(K_{S}^{\otimes l+1}\right)$ (which was equal to the codimension of the image of $\beta_{l}$ in $H^{0}\left(\theta^{\otimes l+1}\right)$ ) is equal to the codimension of $\gamma_{l}$ in $H^{0}\left(B^{\otimes l+1}\right)$. This, together with the claims in (a), (b) and (c) concerning the codimensions of the images of the maps $\alpha_{l}$ proved in (2.7), gives us the codimension of the images of the maps $\gamma_{l}$ in $H^{0}\left(B^{\otimes l+1}\right)$. The claims regarding $\gamma(2,2)$ are proved analogously.

As we did in the case of the canonical ring of regular surfaces of general type, we can characterize when $\bigoplus_{l=0}^{\infty} H^{0}\left(B^{\otimes l}\right)$ is generated in degree less than or equal to 2 using Theorem 4.1 and results from [GP2]:

Corollary 4.2. Let $X$ be a Calabi-Yau threefold with at worst canonical singularities. Let $B$ be a big and base-point-free line bundle on $X$ and let $\varphi$ be the morphism induced by $|B|$. Then $\bigoplus_{l=0}^{\infty} H^{0}\left(B^{\otimes l}\right)$ is generated in degree less than or equal to 2 if and only if

(1) $\varphi$ does not map $X$ generically $2: 1$ onto $\mathbf{P}^{3}$; and

(2) $\varphi(X)$ is not a threefold of minimal degree other than $\mathbf{P}^{3}$.

Proof. Theorem 4.1 tells us that if $\varphi(X)$ is a variety of minimal degree, then the ring $\bigoplus_{l=0}^{\infty} H^{0}\left(B^{\otimes l}\right)$ is generated in degree less than or equal to 2 if and only if $\varphi(X)=\mathbf{P}^{3}$ and the degree of $\varphi$ is greater than 2. If $\varphi(X)$ is not a variety of minimal degree, then in the proofs of [GP2], Theorems 1.4 and 1.7, it is shown that

$$
H^{0}\left(B^{\otimes l}\right) \otimes H^{0}\left(B^{\otimes 1}\right) \stackrel{\gamma_{l}}{\longrightarrow} H^{0}\left(B^{\otimes l+1}\right)
$$

surjects if $l \geq 2$.

The study of the generators of the ring $\bigoplus_{l=0}^{\infty} H^{0}\left(B^{\otimes l}\right)$ is closely related with the question of when $B^{\otimes m}$ is normally generated when $B$ is ample. Recall that a line bundle $L$ is said to be normally generated, or to satisfy property $N_{0}$, if it is very ample and the image of the morphism induced by $|L|$ is a projectively normal variety. This is equivalent to the ring $\bigoplus_{k=0}^{\infty} H^{0}\left(L^{\otimes k}\right)$ being generated in degree 1. In the present context ( $X$ a Calabi-Yau threefold and $B$ an ample and basepoint-free line bundle on $X$ ), the answer to the question of when $B^{\otimes m}$ is normally generated is partially known. If the image of $X$ by the morphism $\varphi$ induced by $|B|$ is not a variety of minimal degree, the authors gave a complete answer to this question for $m \geq 2$ : in this case, in GP2], Corollary 1.1, Theorem 1.4 and Theorem 
1.7 , they proved that $B^{\otimes m}$ is normally generated if $m \geq 2$. The way of proving those results serves to illustrate the relation between the study of the generators of $\bigoplus_{l=0}^{\infty} H^{0}\left(B^{\otimes l}\right)$ and the normal generation of powers of $B$. For instance, in order to prove the normal generation of $B^{\otimes 2}$, the first step is to show that the map

$$
H^{0}\left(B^{\otimes 2}\right) \otimes H^{0}\left(B^{\otimes 2}\right) \longrightarrow H^{0}\left(B^{\otimes 4}\right)
$$

surjects. This was proved in [GP2] by showing that $\bigoplus_{l=0}^{\infty} H^{0}\left(B^{\otimes l}\right)$ is generated in degree less than or equal to 2 and that the map

$$
H^{0}\left(B^{\otimes 3}\right) \otimes H^{0}(B) \longrightarrow H^{0}\left(B^{\otimes 4}\right)
$$

surjects. If $\varphi(X)$ is a variety of minimal degree, the situation is more complex. If $m \geq 3$, the authors also gave in GP2], Corollary 1.1 and Theorem 1.4, a complete answer: $B^{\otimes m}$ is normally generated if and only if $m \geq 4$ or if $m \geq 3$ and $\varphi$ does not map $X 2: 1$ onto linear $\mathbf{P}^{3}$. If $m=2$, answering the question will settle the following

Conjecture 4.3 (cf. GP2, Conjecture 1.9). Let $X$ be a Calabi-Yau threefold and let $B$ be an ample and base-point-free line bundle. Then $B^{\otimes 2}$ satisfies property $N_{0}$ if and only if there is a smooth non-hyperelliptic curve $C$ in $\left|B \otimes \mathcal{O}_{S}\right|$, for some $S \in|B|$.

This conjecture would also give a characterization of when $B^{\otimes 2}$ is very ample. This question of when $B^{\otimes 2}$ is very ample is also open.

One might ask what light the results proved in this section shed on the conjecture. Theorem 4.1 says that if $\varphi(X)$ is $\mathbf{P}^{3}$, then the ring $\bigoplus_{l=0}^{\infty} H^{0}\left(B^{\otimes l}\right)$ is generated in degree less than or equal to 2 if and only if the degree of $\varphi$ is greater than 2. In this case, however, it was seen in the proof of Theorem 4.1 that $\gamma_{3}$ did not surject. On the other hand, if $\varphi(X)$ is a variety of minimal degree different from $\mathbf{P}^{3}, \gamma_{3}$ does surject but $\bigoplus_{l=0}^{\infty} H^{0}\left(B^{\otimes l}\right)$ is not generated in degree less than or equal to 2 . Therefore the strategy outlined before to study the normal generation of $B^{\otimes 2}$, which worked when $\varphi(X)$ was not of minimal degree, does not work if $\varphi(X)$ is of minimal degree. We point out that the conjecture is nevertheless true if $\varphi(X)$ is linear $\mathbf{P}^{3}$ (see GP2, Corollary 1.8). This also follows from the methods of this article, by studying the map $\gamma(2,2)$ in the proof of Theorem 4.1 . Thus the only case left in order to settle the conjecture is when $\varphi(X)$ is a variety of minimal degree different from $\mathbf{P}^{3}$, which should be addressed using a subtler strategy.

The results of Section 3 regarding the structure of the canonical morphisms of regular surfaces of general type onto surfaces of minimal degree has some interesting consequences for Calabi-Yau threefolds. As we will see, Proposition 3.1, Corollary 3.2 and Proposition 3.3 prevent many a priori natural examples of Calabi-Yau threefolds from existing. This also shows that if there do exist examples of prime degree Calabi-Yau covers of threefolds of minimal degree induced by complete linear series, then they cannot come from group actions. We summarize this in the next two corollaries:

Corollary 4.4. Let $X$ be a Calabi-Yau threefold with at worst canonical singularities. Let $B$ be a base-point-free line bundle. Let $X \stackrel{\varphi}{\longrightarrow} Y$ be the morphism induced by the complete linear series $|B|$. Let $n$ be the degree of $\varphi$. Let $U$ be a smooth open set of $Y$ whose complement has codimension 2, and let $L$ be a line bundle on $U$. Assume that

$$
\left.\left(\varphi_{*} \mathcal{O}_{X}\right)\right|_{U}=\mathcal{O}_{U} \oplus L^{-1} \oplus \cdots \oplus L^{\otimes 1-n}
$$


If $Y$ is a variety of minimal degree, then the degree $n=2$ or $n=3$. In particular, if $\varphi$ is a cyclic cover, the degree of $\varphi$ is 2 or 3

Proof. Let $Y^{\prime}$ be a suitable hyperplane section of $Y$, and $S$ the pullback of $Y^{\prime}$ by $\varphi$. Then $\left.\varphi\right|_{S}$ is the canonical morphism of $S$ and satisfies the hypothesis of Proposition 3.1 , and the thesis is clear.

Notation 4.5 . We will call a morphism $\varphi$ satisfying $(*)$ in the statement of Corollary 4.4 a quasi-cyclic cover.

Corollary 4.6. Let $X$ be a Calabi-Yau threefold with at worst canonical singularities. If $X \stackrel{\varphi}{\rightarrow} Y$ is a generically finite morphism onto a smooth scroll $Y \subset \mathbf{P}^{N}$, then the degree of $\varphi$ is even. In particular, there are no generically finite covers of odd degree of smooth rational normal scrolls.

Proof. The proof is analogous to that of Corollary 4.4, using now Proposition 3.3.

In GP2] we described what finite morphisms from a Calabi-Yau threefold onto a variety of minimal degree induced by complete linear series were possible. After Corollary 4.4 and Corollary 4.6 we can obtain the following sharper version of the result in [GP2] (compare with [GP2], Proposition 1.6):

Proposition 4.7. Let $X$ be a smooth Calabi-Yau threefold, let $\varphi$ be the morphism induced by the complete linear series of an ample and base-point-free line bundle $B$ on $X$, and let $n$ be the degree of $\varphi$. If $\varphi(X)$ is a variety of minimal degree, then one of the following occurs:

1) $Y=\mathbf{P}^{3}$ and $n \leq 24$

2) $Y$ is a smooth quadric hypersurface in $\mathbf{P}^{4}$ and $n=2,4,6,8,10,12$ or 14 .

3) $Y$ is a smooth rational normal scroll of dimension 3 in $\mathbf{P}^{5}$ and $n=2,4,6,8,10$ or 12 .

4) $Y$ is a smooth rational normal scroll of dimension 3 in $\mathbf{P}^{N}, N \geq 6, n=2$, and $X$ is fibered over $\mathbf{P}^{1}$ with a smooth $K 3$ surface as a general fiber. The restriction of $B$ to the general fiber of $X$ is hyperelliptic, with sectional genus 2, and its complete linear series maps the fiber $2: 1$ onto a general fiber of the scroll.

5) $Y$ is a smooth rational normal scroll of dimension 3 in $\mathbf{P}^{N}, N \geq 6, n=6$, and $X$ is fibered over $\mathbf{P}^{1}$ with a smooth abelian surface as a general fiber. The restriction of $B$ to the general fiber of $X$ is a $(1,3)$ polarization, and its complete linear series maps the fiber $6: 1$ onto a general fiber of the scroll.

6) $Y$ is a cone over a conic in $\mathbf{P}^{2}$.

7) $Y$ is a cone over a twisted cubic in $\mathbf{P}^{3}$.

8) $Y$ is a cone over a Veronese surface.

In addition, if $X$ has at worst canonical singularities and $\varphi$ is a quasi-cyclic cover, then $n=2$ or 3 .

\section{REFERENCES}

[Bo] E. Bombieri, Canonical models of surfaces of general type, Inst. Hautes Etudes Sci. Publ. Math. 42 (1973), 171-219. MR 47:6710

[Ca] F. Catanese, On the moduli spaces of surfaces of general type, J. Differential Geometry 19 (1984), 483-515. MR 86h:14031

[Ci] C. Ciliberto, Sul grado dei generatori dell'anello di una superficie di tipo generale, Rend. Sem. Mat. Univ. Politec. Torino 41 (1983), 83-111. MR 86d:14036 
[EH] D. Eisenbud and J. Harris, On varieties of minimal degree (a centennial account), Algebraic Geometry, Bowdoin 1985, Amer. Math. Soc. Sympos. in Pure and Appl. Math. 46 (1987), 1-14. MR 89f:14042

[GP1] F. J. Gallego and B. P. Purnaprajna, Projective normality and syzygies of algebraic surfaces, J. Reine Angew. Math. 506 (1999), 145-180. MR 2000a:13023 MR 2001b:13016

[GP2] F. J. Gallego and B. P. Purnaprajna, Very ampleness and higher syzygies for Calabi-Yau threefolds, Math. Ann. 312 (1998), 133-149. MR 99g:14048

[GP3] F. J. Gallego and B. P. Purnaprajna, Canonical covers of varieties of minimal degree, Preprint math.AG/0205010. To appear in "A tribute to Seshadri-a collection of papers on Geometry and Representation Theory", Hindustan Book Agency (India) Ltd.

[GP4] F. J. Gallego and B. P. Purnaprajna, Some homogeneous rings associated to finite morphisms, Preprint. To appear in "Advances in Algebra and Geometry" (Hyderabad Conference 2001), Hindustan Book Agency (India) Ltd.

[GP5] F. J. Gallego and B. P. Purnaprajna, On the rings of trigonal curves, in preparation.

[G] M. L. Green, The canonical ring of a variety of general type, Duke Math. J. 49 (1982), 1087-1113. MR 84k:14006

[HM] D. Hahn and R. Miranda, Quadruple covers of algebraic varieties, J. Algebraic Geom. 8 (1999), 1-30. MR 99k:14028

[H1] E. Horikawa, Algebraic surfaces of general type with small $c_{1}^{2}$. I, Ann. of Math. (2) 104 (1976), 357-387. MR 54:12789

[H2] E. Horikawa, Algebraic surfaces of general type with small $c_{1}^{2}$, II, Invent. Math. 37 (1976), 121-155. MR 57:334

[H3] E. Horikawa, Algebraic surfaces of general type with small $c_{1}^{2}$, III, Invent. Math. 47 (1978), 209-248. MR 80h:14012a

[H4] E. Horikawa, Algebraic surfaces of general type with small $c_{1}^{2}$, IV, Invent. Math. 50 (1978/79), 103-128. MR 80h:14012b

[Kod] K. Kodaira, Pluricanonical systems on algebraic surfaces of general type, J. Math. Soc. Japan 20 (1968), 170-192. MR 37:212

[Kon] K. Konno, Algebraic surfaces of general type with $c_{1}^{2}=3 p_{g}-6$, Math. Ann. 290 (1991), 77-107. MR 92i:14039

[MP] M. Mendes Lopes and R. Pardini, Triple canonical surfaces of minimal degree, International J. Math. 11 (2000), 553-578. MR 2001h:14049

$[\mathrm{M}] \quad$ D. Mumford, Varieties defined by quadratic equations, Corso CIME in Questions on Algebraic Varieties, Edizioni Cremonese, Rome (1970), 29-100. MR 44:209

[OP] K. Oguiso and T. Peternell, On polarized canonical Calabi-Yau threefolds, Math. Ann. 301 (1995), 237-248. MR 96b:14050

[R] M. Reid, Infinitesimal view of extending a hyperplane section-deformation theory and computer algebra, Algebraic geometry, Proceedings L'Aquila 1988, 214-286, Lecture Notes in Math. 1417, Springer-Verlag, Berlin, 1990. MR 91h:14018

Departamento de Álgebra, Facultad de Ciencias Matemáticas, Universidad ComPlutense de Madrid, 28040 Madrid, Spain

E-mail address: FJavier_Gallego@mat.ucm.es

Department of Mathematics, University of Kansas, 405 Snow Hall, Lawrence, Kansas $66045-2142$

E-mail address: purna@math.ukans.edu 